\title{
Research on Ship Power Plant Simulation System Based on LabVIEW and MATLAB Mixed Programming
}

\author{
Jun-rui Jia \\ Department of Mechatronics, Nantong Vocational Technical Shipping College, Nantong \\ 226010, China \\ 594303951@qq.com
}

Key words: Ship power station, LabVIEW, MATLAB, mixed programming, simulation.

\begin{abstract}
Based on the mathematical model of diesel engine, marine generator and load, the simulation operation has been run by MATLAB. The simulation system of ship power station is established by using the mixed programming technology of LabVIEW and MATLAB. The simulation operation results show that the generator output signal is stable and correct, which is of great practical value for the operation analysis of ship power station.
\end{abstract}

\section{Introduction}

A ship's power system is a whole consisting of one or several ship's power sources running under unified monitoring and managing and the connected ship's electrified wire netting, which supplies electric power to the load. The system is also a whole which is connected by the power source device, the power distribution equipment, the electrified wire netting and the load according to the certain way, is the general name of all devices and electric power nets such as the generation, transmission, distribution and consumption of ship power. Therefore, it is of great significance to ensure the safe and reliable operation of the ship's power system. Generally speaking, for the electric propulsion ship, the automatic control system of the ship power station mainly completes the control of the diesel engine speed, output power and the amplitude and frequency of the marine generator output voltage and so on. Based on the characteristics of the ship power system dynamic change, the modeling and simulation of ship power station control system using LabVIEW and MATLAB mixed programming is to establish the ship power plant simulation system[1].

\section{Establishment of Mathematical Model for Ship Power Station System}

\subsection{Simplified Mathematical Model of Diesel Engine}

According to the characteristics of the diesel engine, when the resistance torque changes, the diesel engine will change the fuel supply accordingly, the output torque and the resistance moment will be equal again in order to achieve a new balance. According to Dahlberg principle, the dynamic equations of the diesel engine between the two equilibrium points are as follows:

$$
\Delta T_{o}-\Delta T_{r}+\left(C_{2}-C_{1}\right) * \Delta \omega-I * \Delta \omega=J \frac{d \Delta \omega}{d t}
$$

where ${ }^{\Delta T} T_{\text {o }}$ represents the difference between the output torque of the next equilibrium point and the previous equilibrium point, ${ }^{\Delta T_{r}}$ represents the difference of the resistance moment between the latter and the previous equilibrium point, $\left(C_{2}-C_{1}\right)$ is the load (torque) for the two equilibrium points - The difference between the slope of the speed characteristic curve, $\Delta \omega$ is the diesel engine crankshaft angular velocity, $J$ is the unit inertia, $I$ is the unit damping coefficient.

The change of diesel engine output torque is achieved by changing the fuel supply of diesel engine. In this paper, the actual rack position of oil pump rack and the output torque variable quantity of diesel engine are regarded as the proportional link. ${ }^{K_{4}}$ is defined as the proportional 
coefficient. The transfer function is as follows:

$$
W f(s)=\frac{\Delta T o(s)}{F(s)}=K_{4}
$$

The time delay of the diesel engine is considered as a second-order inertia plus delay in this paper, and its transfer function is:

$$
W d(s)=K p^{*} e^{-T_{3}(s)} /\left[T_{1}(s)+1\right]\left[T_{2}(s)+1\right]
$$

where $K_{p}$ is the amplification factor; $T_{1}$ is the diesel engine generator unit moving parts of the inertia time constant; $T_{2}$ is the gas with the thermal inertia time constant; $T_{3}$ is the diesel engine cylinder pure delay time. Therefore, functions (1), (2), (3) are the simplified diesel engine mathematical models in the speed regulation process[2].

\subsection{Mathematical Model of Diesel Governing System}

In this paper, PID control is used to describe the speed regulation system of diesel generator. In the mathematical model of the PID controller, the controller outputs $m(t)$, the deviation signal is e (t), the transfer function of the controller is as follows:

$$
m(t)=K_{P} e(t)+K_{P}^{T} \frac{d e(t)}{d t}+\frac{K_{P}}{T_{i}} \int e(t) d t
$$

where ${ }^{K} P$ is the proportional gain; ${ }^{T} d$ is the differential time constant; ${ }^{T}$ is the integration time constant.

The following result can be obtained by differential method with (4):

$$
M_{(n)}=K_{P}\left[E_{n}+T_{d} \frac{\Delta E_{(n)}}{T}+\frac{1}{T_{i}} \sum_{J=0}^{n} E(j) T\right]
$$

where $\mathrm{T}$ is the sampling period,

$$
\Delta E_{(n)}=E_{(n)}-E_{(n-1)} \text {. }
$$

For the previous sample,

$$
M_{(n-1)}=K_{P}\left[E_{(n-1)}+T_{d} \frac{\Delta E_{(n-1)}}{T}+\frac{1}{T_{i}} \sum_{J=0}^{n-1} E(j) T\right]
$$

where

$$
\Delta E_{(n-1)}=E_{(n-1)}-E_{(n-2)} .
$$

If the incremental output has the increment, one can obtain the incremental model as follows:

$$
\Delta M_{(n)}=M_{(n)}-M_{(n-1)}=K_{P}\left[E_{(n)}-E_{(n-1)}+T_{d} \frac{\Delta E_{(n)}-\Delta E_{(n-1)}}{T}+\frac{1}{T_{i}} E_{(n)}\right]
$$

Another location model of $\mathrm{m}$ (n) can be obtained by $\Delta M_{(n)}$ plus $M_{(n-1)}$

$$
M_{(n)}=\Delta M_{(n)}+M_{(n-1)}=M_{(n-1)}+P_{0} E_{(n)}+P_{1} E_{(n-1)}+P_{2} E_{(n-2)}
$$
where

$$
P_{0}=K_{P}\left(1+\frac{T_{d}}{T}+\frac{T}{T_{i}}\right) \quad P_{1}=K_{P}\left(1+\frac{2 T_{d}}{T}\right) \quad P_{2}=K_{P} \frac{T_{d}}{T} .
$$

Therefore, the PID controller simulation model is described as equation (8) in this paper.

\subsection{Mathematical Model of Synchronous Generator}

In modern ships, all marine generators used three-phase AC synchronous generator as the main power supply equipment. With the large-scale development of the ship, the single generator capacity of the marine generator is continuously increasing. In order to ensure the stable operation of the ship power system, it puts forward demand for the reliability of the generator carrier frequency control system.

Consider the third-order mathematical model of marine generator is simple, reliable and easy to use in the dynamic analysis of marine power system, and it can take into account the dynamic 
changes of excitation system. Therefore, the third-order ship generator model is used as the simulator of ship power control system in this paper. The model is a transient equation in the ${ }^{d}{ }_{q o}$ coordinate, widely used in power system analysis which is not required by precision, but still needs to take account of dynamic system of excitation system[3].

$$
\begin{gathered}
u_{d}=x_{q} i_{q}-r_{a} i_{d} \\
u_{q}=E_{q}^{\prime}-x_{d}^{\prime} i_{d}-r_{a} i_{q} \\
T_{d 0} p E_{q}^{\prime}=E_{f}-E_{q}^{\prime}-\left(x_{d}-x_{d}^{\prime}\right) i_{d} \\
T_{j} \frac{d_{\omega}}{d_{t}}=T_{m}-T_{e}=T_{m}-\left[E_{q}^{\prime} i_{q}-\left(x_{d}-x_{d}^{\prime}\right) i_{d} i_{q}\right] \\
\frac{d_{\delta}}{d_{t}}=\omega-1
\end{gathered}
$$

where $E_{q}^{\prime}, \omega, \sigma$ are the state variables. The q-axis transient electromotive force of the motor is proportional to the flux of the f-winding. Since the flux linkage does not change suddenly during the transient process, the disturbance occurs at $\mathrm{t}=0$, then $E_{q}^{\prime} / t=0+=E_{q}^{\prime} / t=0-$. Thus, the initial value of $E_{q}^{\prime}$ at the time of disturbance can be determined according to the value at steady state, as shown in equation (14).

$$
E_{q o}^{\prime}=u_{q o}+x_{d}^{\prime} i_{d o}+r_{a} i_{q o}
$$

When the excitation system and the governor dynamics are taken into account, ${ }^{E} f$ (the excitation electromotive force) and $T_{m}$ (the mechanical torque)are generally the state quantities of the corresponding system, and the initial value of the disturbance is determined by the steady state value, as shown in equations (15) and (16).

$$
\begin{gathered}
E_{f o}=E_{q o}=E_{q o}^{\prime}+\left(x_{d}-x_{d}{ }^{\prime}\right) i_{d o} \\
T_{m o}=T_{e o}=E_{q o}^{\prime} i_{q o}-\left(x_{d}{ }^{\prime}-x_{q}\right) i_{d o} i_{q o}
\end{gathered}
$$

If the transient state of the stator is neglected in the third-order model, then $p \varphi_{d}=p \varphi_{q}=0$, the aperiodic component of the stator flux linkage and its corresponding pulsating torque will be neglected in the analysis and calculation, which greatly simplifies the analysis.

\subsection{Mathematical Model of Excitation Control System}

In the ship electric power system, the excitation control is the indispensable link of the generator operation. There are two basic types of synchronous generator excitation power: self excited generator and separately excited generator, where the armature winding (or auxiliary winding) of the generator is the self excited generator. The excitation system leads DC which is produced by AC generator into excitation winding through the diode rectifier. Self excited generators are usually initially excited and pressed by the remnant magnetic. Where there is a special excitation power called the separately excited generator. It is usually a small capacity DC or AC exciter coaxial with the generator. Most of the marine generator with rotary-type small synchronous generator as the exciter, which eliminates the slip ring and brush mechanism, it is called brushless synchronous generator. Since there is no brush mechanism, the reliability of the operation is improved and the burden of maintenance is reduced[4]. The quality of the output power quality of the ship's power grid, such as output voltage accuracy, voltage waveform, voltage regulation range and load voltage stability, are determined by the generator excitation system. A 
typical excitation control system is used as the mathematical model in this paper. The transfer function block diagram is shown in Figure 1.

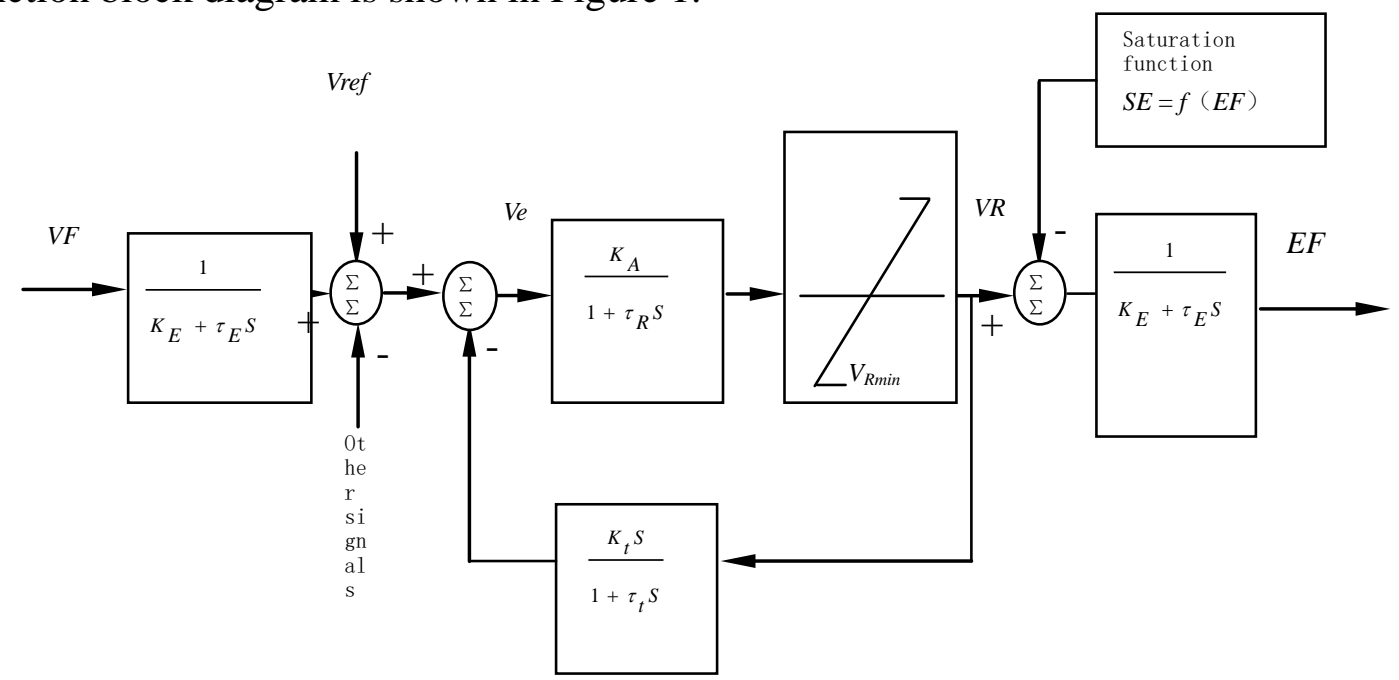

Fig.1 Transfer function diagram of excitation control system

In Fig. $1, V_{F}$ is the generator terminal voltage, Vref is the reference voltage, $V R$ is the exciter input voltage, $K A 、 K E 、 K t 、 \tau R 、 \tau E 、 \tau t$ are constants.

\subsection{Load mathematical model}

The first-order link is used to simulate the load change in this paper, as shown in equations (17) and (18):

$$
\begin{aligned}
\frac{d}{d t} i_{d} & =-\frac{r_{C}}{x_{C}} i_{d}+i_{q}+\frac{1}{x_{C}} u_{d} \\
\frac{d}{d t} i_{q} & =-i_{d}-\frac{r_{C}}{x_{C}} i_{q}+\frac{1}{x_{C}} u_{q}
\end{aligned}
$$

\section{Simulation system development}

\subsection{MATLAB simulation}

In the Simulink interactive simulation environment of MATLAB, the PSB(Power System Blockset) is used to set up the simulation control system of ship power plant. The model is shown in Fig.2.Two marine generators simultaneously supply power for the load through the main power distribution screen. The system measures and simulates voltage waveform, rotor speed and output power in the process of generator running, the output results are respectively shown in Figure $3,4,5$.The results show that the simulation control system of the ship power station established in this paper provides low speed generators and good output three-phase voltage waveforms[5,6]. On this basis, it is of practical significance for us to do sudden, unexpected further unloading load operation analysis and fault simulation experiments with ship power station[7,8]. 


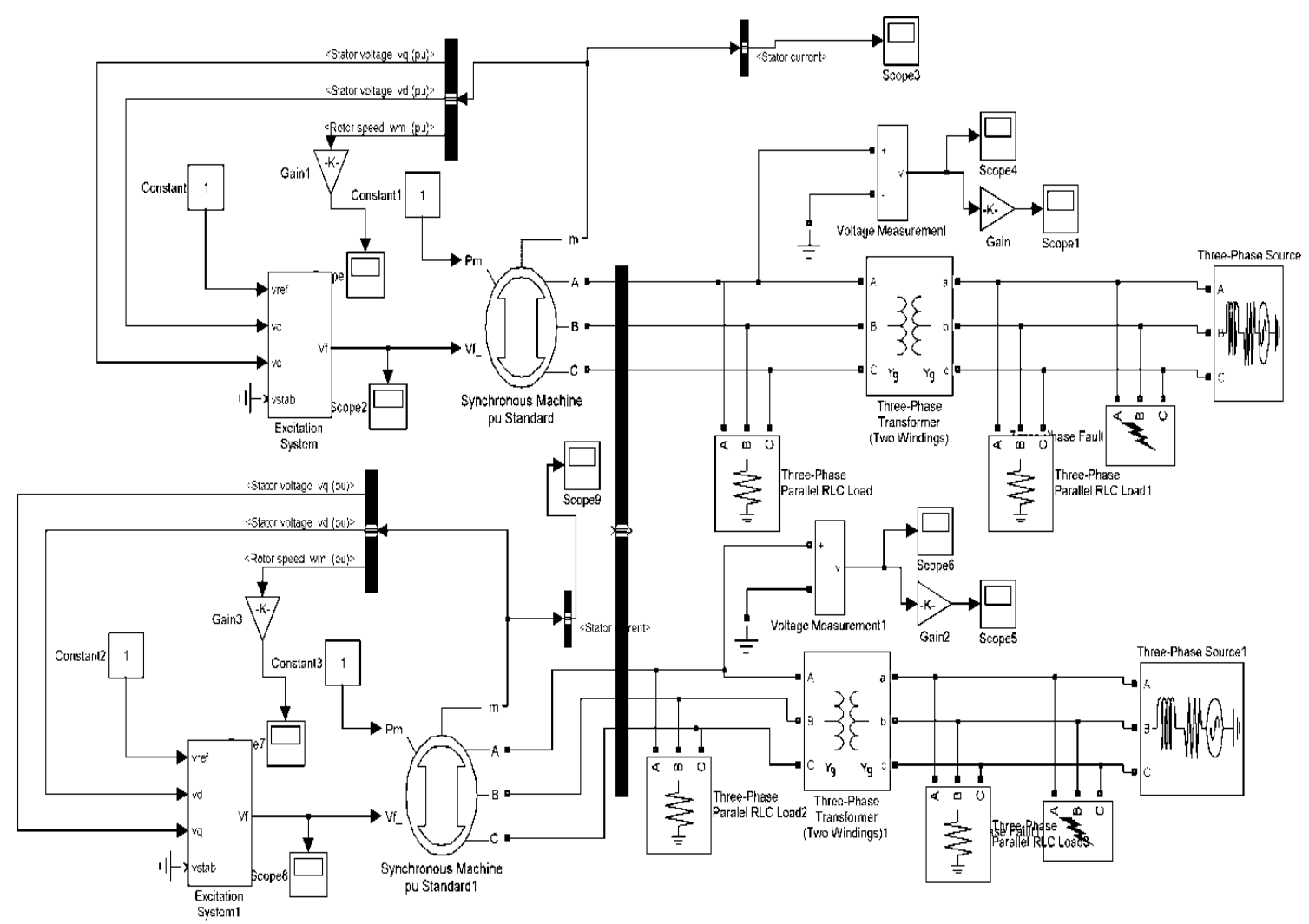

Fig.2 Ship Power Plant Simulation Control System

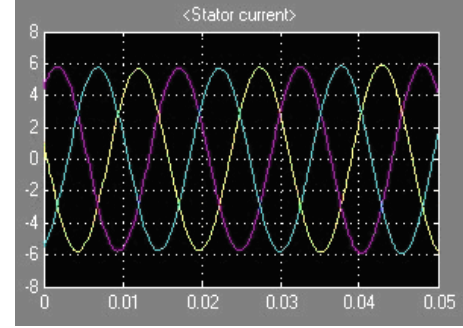

Fig.3 Generator output voltage waveform

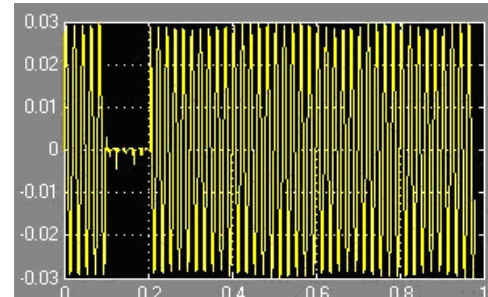

Fig.4 Generator speed change rate curve

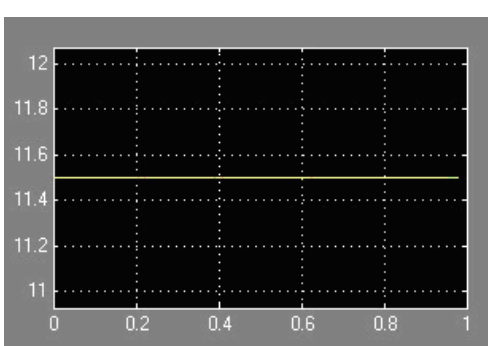

Fig.5 Generator output power

\subsection{Mixed Programming of LabVIEW and MATLAB}

In view of the advantages of both LabVIEW and MATLAB, LabVIEW and MATLAB are 
often mixed programming through powerful external interface of LabVIEW in the process of virtual instrument development, which can improve the system, give full play to the advantages of LabVIEW and MATLAB and rapidly develop powerful intelligent virtual instrument[9-10]. In the process of developing virtual instrument by mixed programming, LabVIEW is usually used to design the user graphical interface, collect data, control hardware, operate network communication. LabVIEW communicates with MATLAB by MATLAB Script node. MATLAB provides neural networks, wavelet analysis and other complex algorithms for LabVIEW in the background[11-12]. In this paper, the monitoring screen of the ship power station simulation system built in LabVIEW by calling the mathematical model of the ship power station simulation system established in MATLAB is shown in Figure 6.

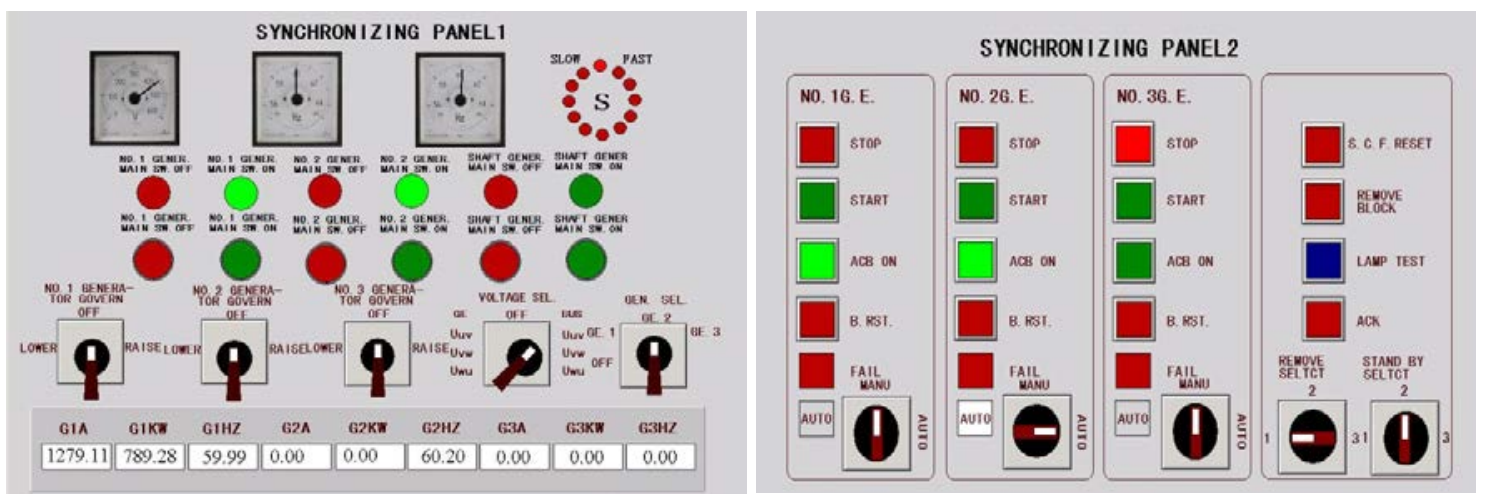

Fig.6 Ship power station simulation system monitoring screen

\section{Conclusion}

On the basis of building main mathematical model of the ship power station system, the simulation model of ship power station control system is established by using the mixed programming of LabVIEW and MATLAB, which gives full play to integral superiority of the two programming software. The system is to simulate the operation of a generator and a standby generator. And the simulation results show that the generator output signal has good stability and can meet the needs of dynamic and static operation analysis of marine power plant. Besides, it can also be used to further study the control of vehicle power station and the automatic regulation of carrier frequency so as to realize the system evaluation and operation analysis of the ship automation power station.

\section{Acknowledgments}

This work was financially supported by Nantong Science and Technology Project Fund in 2015.The title of project is "Design of Electric Bicycle Wireless Charging System Based on Solar Power”.

\section{Reference:}

[1] L.Q. Huang, G.H. Yang, W.N. Yu, Using the Programmable Logic Controller to Realize the Frequency and Power Automatic Control of Ship Power Station. Navigation of China. 2002(3)64-66.

[2] F. Zhu, Y.Z. Xiao, PLC control system for automatic combined engine with quasi-synchronization in ship power plant. Marine technology. 2006(3)28-30.

[3]F.G. Zheng, K. Lee, Marine automation, Dalian maritime university, Dalian, 2010.

[4]Z.X. Chen, S.N. Yang, Implementation of serial communication between virtual instrument and PLC, Automation \& Instrumentation. 2006 (6)42-45.

[5]Y.H. Chou, Q.P. Zhang, J. Lee, Power distribution and regulation of parallel synchronous generator, Marine electric technology. 2005(2)36-38. 
[6]D.Q. Liu, Modeling and simulation of semi-submersible electric propulsion system, Wuhan university of technology school of energy and power engineering, Wuhan, 2009.

[7]P.F. Dai, S.K. Wang, G.F. Wang, Testing engineering and LabVIEW application, Electronic industry, Beijing, 2009.

[8]C. Yang, Design of remote monitoring and diagnosis virtual system for large machinery and equipment, Dalian university of technology, Dalian, 2008.

[9]Z.Y. Lin, Application of LabVIEW in the development of relay protection fault information processing system, Zhejiang University, Zhejiang, 2006.

[10]L. Wang, M. Tao, Proficient in LabVIEW 8.0, Electronic industry, Beijing, 2010.

[11]W.F. Shi, A software technology of data acquisition system for marine power station simulator: submitted to journal of Shanghai marine university. (2001)

[12]H.Y. Zhen, Large container ship marine engine room simulator based on networking technology: submitted to journal of system simulation. (2001) 